\title{
Knowledge Management Capability Framework
}

\author{
Birinder Sandhawalia ${ }^{1}$ and Darren Dalcher ${ }^{2}$ \\ ${ }^{1}$ School of Computing Science, Middlesex University, UK, b.sandhawalia@mdx.ac.uk \\ ${ }^{2}$ School of Computing Science, Middlesex University, UK, d.dalcher@mdx.ac.uk
}

\begin{abstract}
This paper presents a Knowledge Management Capability framework based upon an empirical case study conducted at a CMM Level 5 software project organisation. The paper discusses the development of the organisation's knowledge management (KM) initiative from its initial state, to an organisational state where the KM practices are institutionalised and embedded within the daily activities and work methods of the organisation. The organisation's KM initiative is analysed through the development of two KM capabilities, namely infrastructure and processes, which were examined in depth while conducting the case study, and form the basis for the KM Capability Framework. The resulting framework helps organisations to analyse any imbalance that may exist in their KM initiative and needs to be addressed. In doing so, the framework benefits organisations in making corrections and restoring balance between their KM infrastructure and process capabilities, thereby improving the path of successful KM implementation towards a state of organisational KM capability.
\end{abstract}

Keywords: Knowledge, Knowledge management (KM), Knowledge management capabilities, Knowledge management infrastructure, Knowledge management processes, Knowledge Management Capability Framework

\section{Introduction}

Software project organisations need to leverage their existing knowledge and create new knowledge to be able to innovate and compete effectively. In order to achieve this, organisations must develop the ability to facilitate the flow of knowledge within the development processes of their software projects (Styhre 2003). This research conducted an in-depth case study of a CMM Level 5 software organisation, named XYZ, to identify and analyse the key knowledge management infrastructure and processes required to support and facilitate the flow of knowledge across projects within the organisation. A CMM Level 5 certification was considered important and relevant to ensure that the organisation practiced mature software development processes. Gold et al (2001) and Khalifa and Liu (2003) include leadership, top management support, knowledge culture, and IT 
capability in the form of repositories, asset libraries, intranet portal and collaborative technology as knowledge infrastructure. Alavi and Leidner (2001) list knowledge creation, storage, retrieval, transfer and application as knowledge processes. While conducting the case study, the researcher observed and examined how these knowledge processes manifested in the form of training, mentorship, interaction, feedback, collaboration and application while developing software at XYZ.

The knowledge management initiative at XYZ started as a concept and is now developing into a state where knowledge management practices are being increasingly institutionalised and embedded into the daily work practices and methods of the organisation. For a knowledge management initiative to achieve such an organisational state, the knowledge infrastructure and process capabilities also need to develop from an initial state of low availability, accessibility, usage and practice to a state of organisational capability of high availability, accessibility, usage and practice (Gold et al 2001, Khalifa and Liu 2003). This research adopts KM infrastructure and processes as two dimensions of KM capabilities, and the following sections explain the rationale for adopting them to analyse development of KM capabilities.

\section{KM Infrastructure Capabilities}

Gold et al (2001) identify information technology, organisational structure, and culture as infrastructure capabilities, and acquisition, conversion, application and protection as process capabilities. Khalifa and Liu (2003), while advancing Gold et al's (2001) proposition, establish leadership, culture and KM strategy as infrastructure required to develop a knowledge management initiative.

Information technology is an infrastructure capability as it facilitates knowledge flow and eliminates barriers to communication within an organisation. A flexible organisational structure encourages knowledge sharing and collaboration across boundaries within the organisation, while a rigid structure often has the unintended consequence of inhibiting such practices. Organisational structure capability for facilitating knowledge flow is also shaped by the organisation's policies, processes, and system of rewards and incentives, which determine the channels from which knowledge is accessed and how it flows (Leonard 1995). An organisation's culture is central to encourage interaction and collaboration between individuals that are important to facilitate knowledge flow, and also provides individuals the ability to self-organise their own knowledge and practice networks to facilitate solutions for problems and share knowledge (O'Dell and Grayson 1998). Organisational vision, mission and values embody the culture of the organisation and determine the types of knowledge that are desired and the types of knowledge related activities that are encouraged (Leonard 1995). Leadership sets the overall concept and implementation plan for the knowledge management initiative and obtains commitment from individuals to achieve the desired objectives and outcome. The KM leader helps create the appropriate culture to accomplish the knowledge vision and strategy of the organisation. The 
knowledge management strategy identifies the knowledge requirements and how they are to be fulfilled in congruence with the strategic goals of the organisation.

\section{KM Process Capabilities}

The knowledge management processes of an organisation are focused towards obtaining, sharing, storing, and using knowledge. Examples of these aspects of knowledge management processes within literature are: capture, transfer, and use (DeLong 1997); acquire, collaborate, integrate, experiment (Leonard-Barton 1995); create, transfer, assemble, integrate, and exploit (Teece 1998); create, transfer, use (Spender 1996, Skyrme and Amidon 1998); create, process (Ivers 1998); create, store; transfer and apply (Alavi and Leidner 2001); acquire, convert, apply, protect (Gold et al 2001). An examination of the characteristics of knowledge process capabilities enable them to be grouped into the four broad dimensions of knowledge creation, conversion, transfer and application.

Knowledge creation, as suggested by Nonaka and Takeuchi's (1995) SECI model, is enabled by the processes and activities of interaction, feedback, innovation, brainstorming, and benchmarking. Knowledge conversion (Nonaka and Takeuchi 1995) is made possible through the processes and activities of synthesising, refinement, integration, combination, coordination, distribution and restructuring of knowledge. Shared contexts and common representation are required for knowledge conversion, and mechanisms for facilitating the same are group problem solving and decision-making. Information technologies like email, repositories, intranet portal, teleconferencing, and the activities of mentoring, collaboration and training play a key role in transferring knowledge. Forums such as communities of practice (Wenger and Snyder 2000) and centres of excellence, and training provide a platform for the transfer of knowledge. Knowledge is effectively applied during the developmental processes of an organisation through rules and directives, routines and self-organised teams. Knowledge is applied to formulate and refine the standards, procedures and processes developed to execute tasks within the organisation.

\section{Development of KM Capabilities at XYZ}

The above knowledge processes are dynamic and highly interdependent and intertwined. At any point of time and in any part of an organisation, individuals and teams maybe engaged in several different aspects of these knowledge processes. The main focus of the knowledge processes is to facilitate the flow of knowledge between individuals, and consequently teams, and the major challenge for any knowledge management initiative is to facilitate these flows so that the maximum amount of transfer occurs. Styhre (2003) views knowledge as what emerges in the notion of knowing within a "processual perspective of knowledge that conceives of knowledge as both what is manifested in practices and simultaneously endowed within a conceptual framework." Styhre (2003) states 
that knowledge exists throughout an organisation and is not a clearly bounded and manageable resource that can be located in one single point in time and space. In other words, knowledge is fluid and emergent, and not fixed and stable, and being fluid and moving, it is embedded in social relationships, and emerges in practices and the use of concepts.

In order to make knowledge available throughout an organisation, knowledge management process capability needs be fully leveraged, and this is not possible without the presence of knowledge management infrastructure capability. Gold et al (2001) state that "the presence of both knowledge management process and infrastructural capabilities is critical to reach the intended knowledge management objectives." Appropriate knowledge management infrastructure needs to be implemented to routinise knowledge management processes and practice and to enhance knowledge application in daily business procedures, Grant (1996).

As organisations implement knowledge management initiatives, the knowledge management infrastructure and processes develop. One might expect the development of these knowledge management infrastructure and processes would progress smoothly and in congruence with each other, from an initial state to an organisational state where the KM capabilities are embedded in the daily activities and work practices of the organisation. The path of such an ideal development is represented in Figure 1 where KM infrastructure capability development is represented on the $y$-axis and KM process capability is represented along the $x$ axis of the graph and both capabilities progress from low to high along their respective axis. The ideal, congruent development of both capabilities is represented by arrow $q$, which depicts a smooth progress from an initial to an organisational state. 


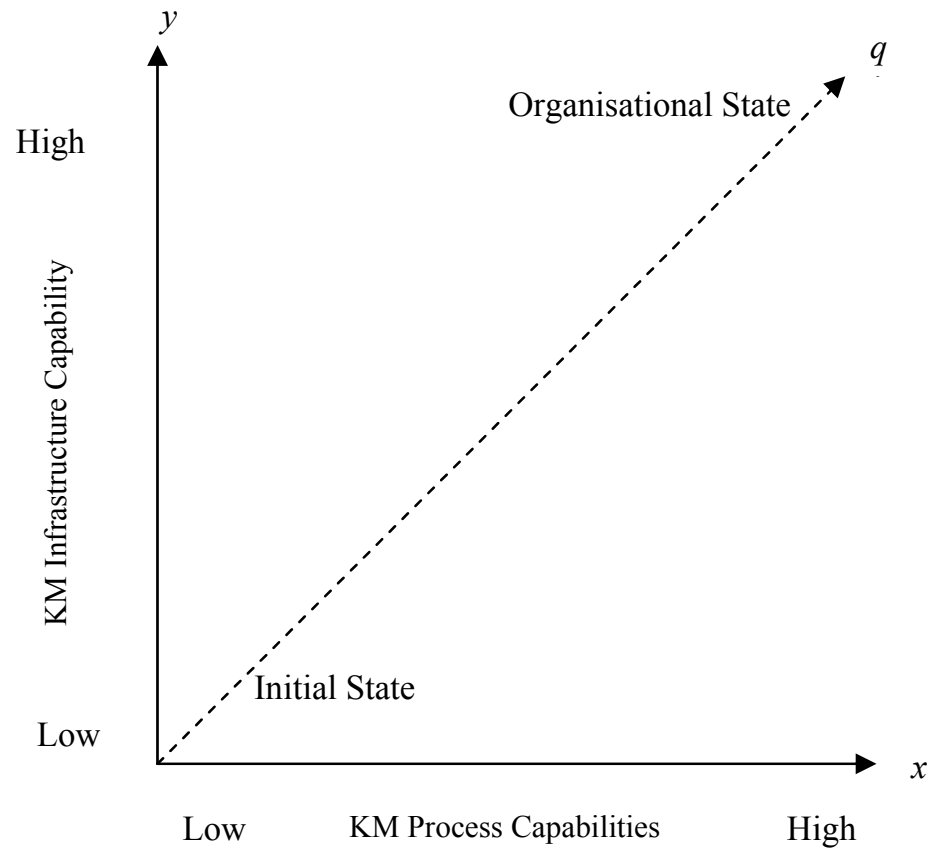

Figure 1. KM Capabilities

\subsection{The Initial State}

However, the research observed that in actual practice at XYZ the path taken during the development of $\mathrm{KM}$ capabilities was not smooth and ideal, as represented by arrow $q$. The $\mathrm{KM}$ initiative at XYZ evolved from the initial practice of documents stored in physical libraries. Individuals were, perhaps unknowingly, performing knowledge process activities while referring to these documents and past project data, and interacting with colleagues. While XYZ always possessed leadership, the organisational structure, culture, vision, and use of collaborative technology also evolved over a period of time. The creation of a central repository marked the beginning of a determined effort to harness the use of technology to improve the efficiency and productivity of existing and future projects. The realisation of the benefits of such efforts motivated senior management at XYZ to explore further possibilities and create a knowledge vision, thus signifying the initial state of development of $\mathrm{KM}$ infrastructure and process capabilities. During this stage XYZ defined what KM meant to it as an organisation, and made clear the concepts and objectives that it wanted to achieve by implementing a KM initiative. A KM strategy was developed ensuring that it 
was connected to other organisational needs and initiatives that already existed, and resources and infrastructure required to implement the initiative were identified.

Thereafter, XYZ started to develop the KM initiative, and consequently the infrastructure and process capabilities. The knowledge vision was translated into action by means of mission and value statements to encourage the growth of knowledge within the organisation. A knowledge culture of sharing was promoted and individuals encouraged to contribute, while project managers were expected to lead their teams in a learning environment of openness, trust and feedback. The introduction of collaborative technology was viewed as a significant step towards establishing the knowledge culture and to a certain extent a change in the organisational structure. The use of email, teleconferencing and bulletin boards were expected to promote collaboration and boundary crossing within department and development centres and hence reduce the silo effect of a previously more vertical structure. A central repository was developed to store process assets and process improvement proposals, while the intranet portal was developed to provide organisation wide dissemination of explicit knowledge (Polanyi 1967) and tools such as IPMS, EKMS, HRS, and CRM. Training was imparted to introduce and make individuals explicitly familiar with these knowledge infrastructure and capabilities. Knowledge sharing activities were made mandatory within the training programmes. However the emphasis on developing KM infrastructure capability while still providing training in knowledge process capability, resulted in high availability of this infrastructure to individuals within the organisation and is represented by arrow $i$ in Figure 2 Arrow $i$ depicts the actual progress of XYZ's KM initiative development, contrary to the expectation depicted in Figure 1, from a state of low infrastructure and process capability to a state where the emphasis on infrastructure capability development was greater than the practice of knowledge process capability. In other words, this state was characterised with a high availability and accessibility of infrastructure capability for individuals compared to the extent to which they were performing KM processes. 


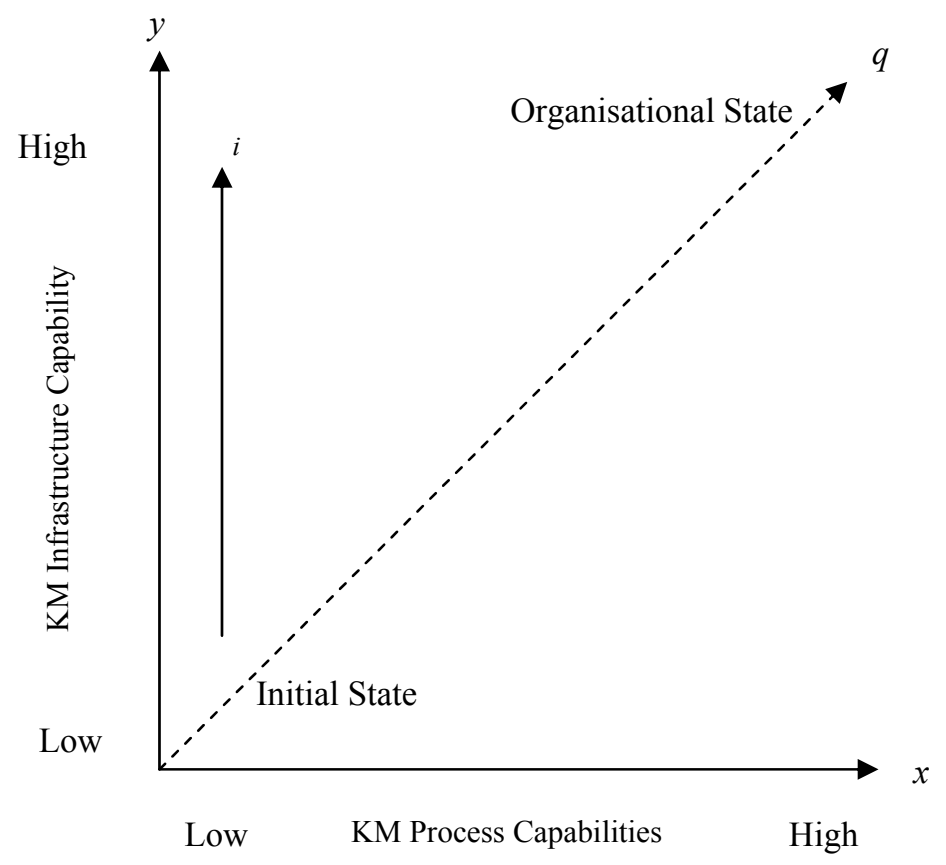

Figure 2. KM Infrastructure Development

\subsection{The Deviation}

When this researcher first visited and commenced research activities, the number of individuals employed by XYZ was 50,000. Thereafter, the researcher made numerous visits to $X Y Z$ over the course of the next two years by when the number of individuals employed by XYZ was 85,000. A senior Project Manager at XYZ stated that "fifty percent of our new employees have been at XYZ for less than three years." The rapid increase in the number of new employees was representative of XYZ's expansion strategy which was characterised with the acquisition and opening of development and delivery centres across the globe. This resulted in XYZ becoming a larger global organisation with employees from diverse background and cultures working in a more distributed environment. A small number of new employees were recruited as part of XYZ's strategy to employ "bench strength that would provide a bigger talent pool." The idea of employing 'bench strength' was that XYZ would provide individuals ongoing training and therefore have reserve skilled employee resources for job rotation, cover for absentees and starting new projects. However, the number of individuals 
employed as 'bench strength' was less that five percent of the total number of new employees.

The above mentioned rapid expansion had an effect on XYZ's KM initiative, and the infrastructure and process capabilities. New employees were provided with training to perform knowledge process capabilities as a part of their induction programme. However, when they were assigned to projects upon completing their training, the infrastructure capability proved inadequate and insufficient. There was a loss of knowledge richness due to the distributed and less face-to-face knowledge, and the knowledge assets were considered to be scattered. There was a perceived lack of 'teamness' that resulted in a coordination breakdown in project management activities. XYZ had to address cultural differences amongst globally distributed employees who had to adjust to new work practices. While the new employees' initial training helped overcome some of these issues and inculcated knowledge processes, the infrastructure capabilities of organisational structure, culture, information technology and KM strategy needed to be reassessed and improved upon. This resulted in XYZ's possessing inadequate KM infrastructure capability compared to the number of employees seeking to perform KM process capabilities. The progression to this state is depicted in Figure 3 by arrow $p$ from the previous state of high infrastructure capability and low process capability, to a new state of low infrastructure capability and high process capability. 


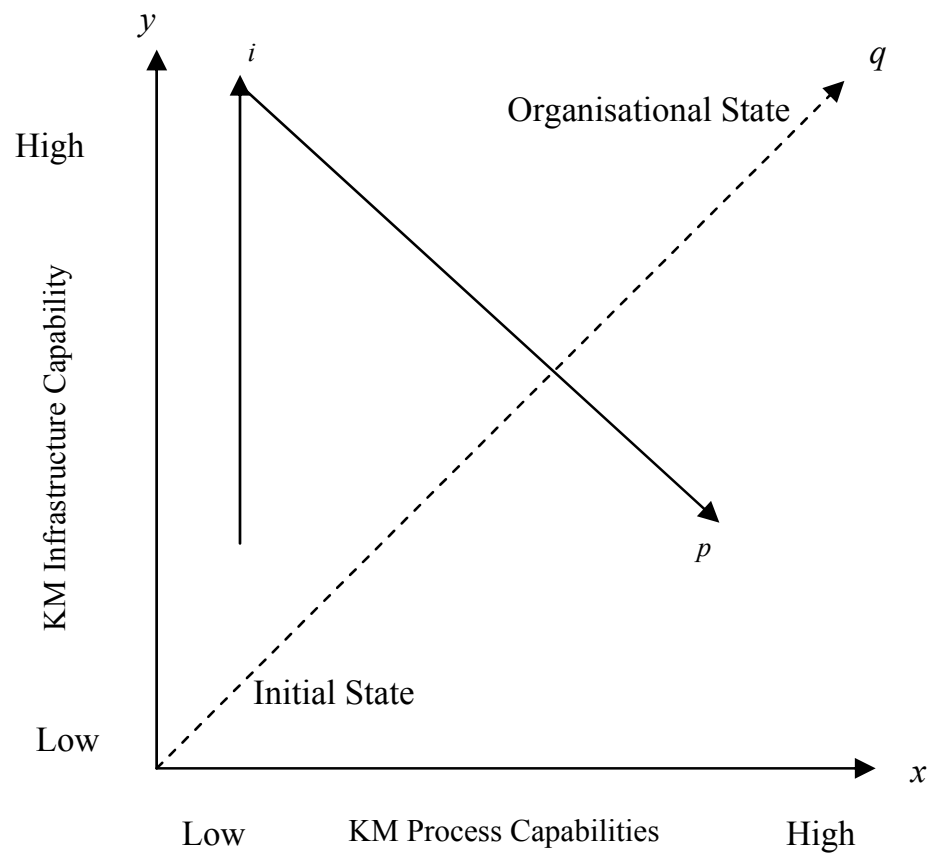

Figure 3. KM Process Capability Development

\subsection{The Correction}

Having recognised the problem, XYZ addressed the issues presented by the state of low infrastructure capability and high process capability by increasing site visits and travel of individuals amongst different development and delivery centres, having local, acculturated knowledge champions and interaction among them at the regional and corporate level, encouraging regional and virtual communities of practice, and also starting regional and corporate centres of excellence. XYZ attempted to create a combination of both "top-down and bottom-up knowledge culture." The knowledge champions were made responsible of ensuring that knowledge created at the global development and delivery centres, was made available to the local or regional centres and the overall knowledge owner at the corporate level. The existing EKMS was upgraded to a new knowledge management system (KMS) which as mentioned by a senior group lead during an interview, "consolidated all scattered knowledge assets into one system that caters to the global needs of 85,000 diverse employees." The people knowledge map was introduced as an integral part of the upgraded KMS to help identify experts and individuals with experience for projects with specific characteristics. The upgraded KMS was implemented, and along with the other measures 
mentioned above, was expected to be a catalyst that drives knowledge flow, and progresses $\mathrm{XYZ}$ to an organisational state of high infrastructure and process capability, where KM practices are institutionalised and embedded in the daily activities and processes of the organisation. This progress of XYZ's KM initiative from a state of low infrastructure capability and high process capability to a state high infrastructure and process capability is depicted by arrow $e$ in Figure 4 completing the $\mathrm{N}$-shaped journey to a higher organisational state in contract with the initial expectation of smooth transition.

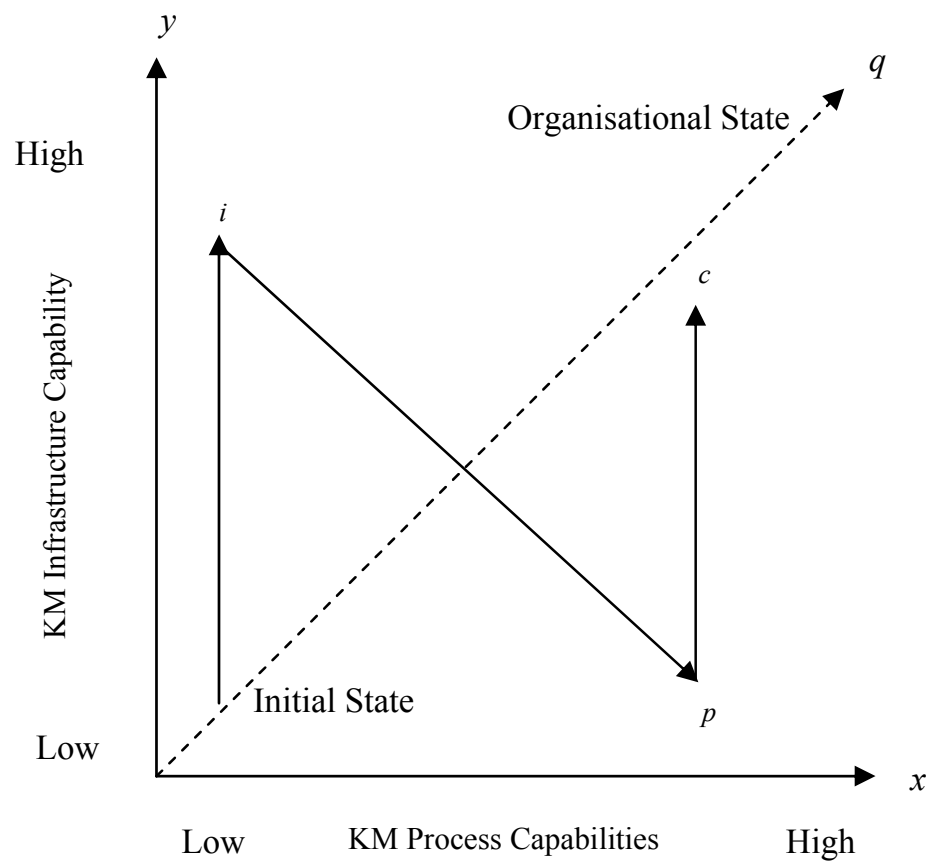

Figure 4. Towards an Organisational State of KM Capability Development

\subsection{Case Study Summary}

The implementation of the KM initiative at XYZ provides an example of how organisations need to balance the growth and development of KM processes and infrastructure while developing their KM programmes. Organisations expect a smooth path from conceptualising a KM initiative to its successful implementation. XYZ's experience highlights two stages within the implementation of its KM initiative when an imbalance existed between the KM infrastructure and process capabilities. When XYZ was developing the concept of 
the KM initiative after its initial conceptual stage, the organisation put an emphasis on developing the infrastructure. This resulted in greater availability of $\mathrm{KM}$ infrastructure capability than $\mathrm{KM}$ processes being practiced, even though training was introduced for these processes thereby representing a state of higher $\mathrm{KM}$ infrastructure capability and lesser KM process capability. Thereafter, with the addition of a number of employees and their training at induction, the KM infrastructure capability was inadequate to support the KM processes practiced by the individuals. This represented a state of greater KM processes being practiced and lesser KM infrastructure capability being available. XYZ started progressing towards a state of organisational KM capability after it addressed these imbalances.

\section{KM Capability Framework}

The above discussion highlights the issues faced by XYZ while developing a knowledge management initiative in order to mobilise and utilise its knowledge resources. While the findings pertain to a single organisation, they may also reflect the view of Eskerod and Skriver (2007) who studied the literature on knowledge transfer and identified persistent issues that impact such efforts suggesting a more general trend. Eskerod and Skriver (2007) state that "however.....many companies experience serious problems when trying to make knowledge transfer work." In the case of XYZ, the organisation struggled to make knowledge resources available to all individuals when it inducted a significant number of new employees. This problem is made apparent by the downward arrow $p$ in Figures 3 and 4 when the KM infrastructure was found inadequate to support the knowledge needs of a larger number of organisational individuals. Thus Eskerod and Skriver's (2007) view helps understand the phenomenon observed at XYZ.

The discussion in Section 4 confirms that if an organisation conceptualises its $\mathrm{KM}$ programme in an initial state and intends to achieve an organisational state where the KM capabilities are institutionalised and embedded within the organisation's daily procedures, processes and practices, two other intermediate and distinct capability states also exist. One state is of higher KM infrastructure capability availability and lesser KM process being practiced, while the other state is of greater KM processes being practiced and lesser KM infrastructure capability being available. The four states are represented in Figure 5. Also represented in the figure is the ideal path an organisation would expect to progress along when launching a KM programme, and indirectly the possible paths along which their KM programme might progress during implementation. The path to implementing a KM programme does not progress directly and smoothly from the initial to organisational state as envisaged by $\mathrm{XYZ}$, but might instead progress through either of the two intermediate states, or as in XYZ's case through both intermediate states. If an organisation initially lays more emphasis on developing its $\mathrm{KM}$ infrastructure capability it will progress to the state of higher KM infrastructure capability and lesser process capability before it can progress to an 
organisational state. On the other hand, if the organisation was to initially lay more emphasis on practicing KM processes it will progress to the state of greater process capability and lesser infrastructure capability and before being able to progress towards the organisational state. However, as XYZ's experience depicted, a large organisation could progress from one intermediate state to another before progressing towards the organisational state of KM capability. This is a very important observation because many organisations tend to launch knowledge management programmes without due consideration of the organisation's capabilities to guarantee any measure of success of implementation (Davenport et al 1998, Leonard 1995). As the case study evidence revealed, even one of the largest software project organisation with CMM Level 5 accreditation needs to coordinate its KM capability development to achieve a state of organisational knowledge management.

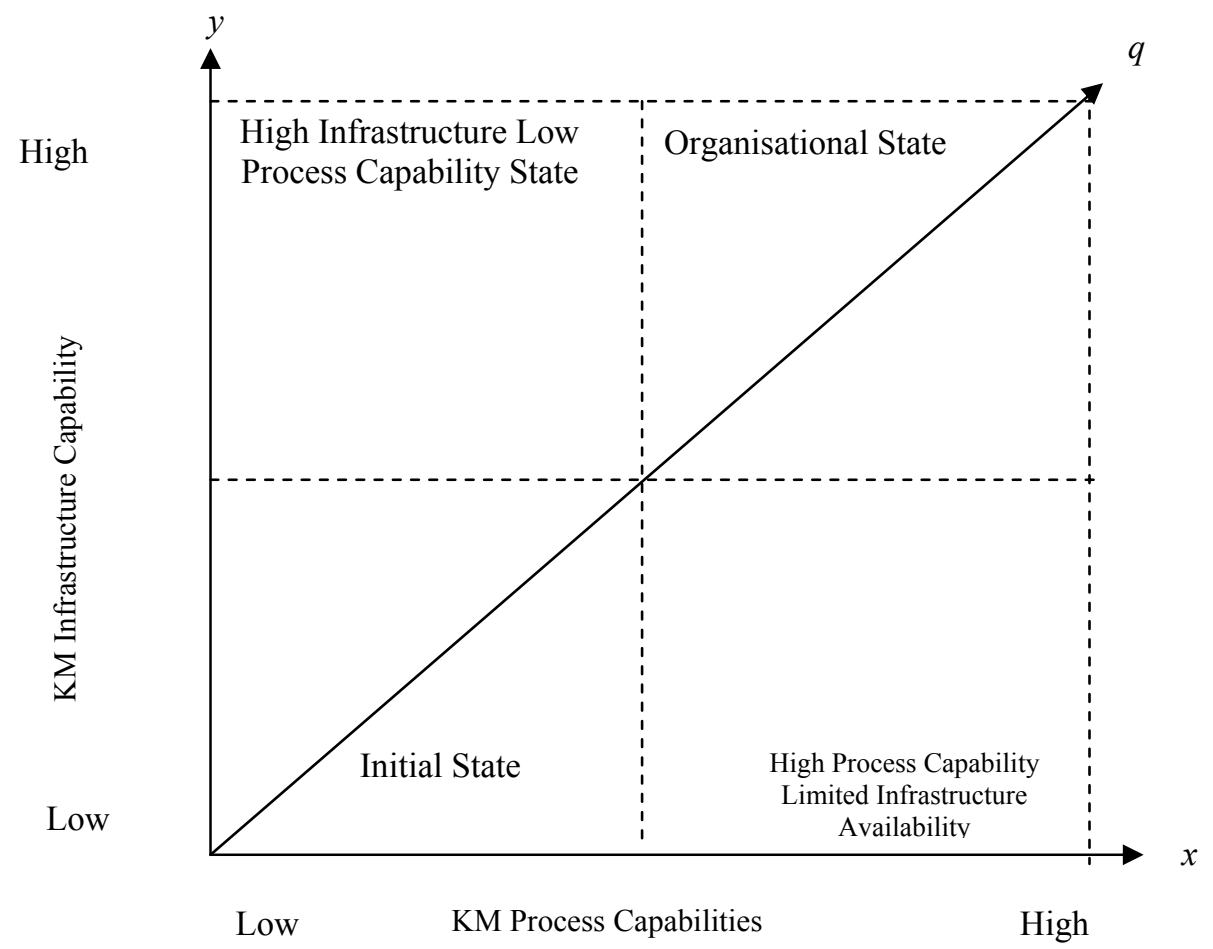

Figure 5. KM Capability Framework

The framework presented in Figure 5 depicts the possible states organisations may progress along while implementing their KM programmes. Organisations can benefit by referring to the framework to determine the progress of their $\mathrm{KM}$ programmes. The characteristics of each state described below will help organisations identify the current state of their knowledge management programmes. 


\subsection{Initial State}

An organisation's KM programme can be considered to be in the initial state when the organisation is creating a knowledge vision and relating this vision to its strategic needs and other initiatives that already exist. During this state the organisation explores all possibilities related to the $\mathrm{KM}$ initiative and also the opportunities present. The organisation identifies the infrastructure required to support the initiative and the KM processes to be practiced. Financial support for the programme and other resources required to implement the programme are also identified and budgeted. An important activity or feature of this state is the top management's commitment to the KM initiative and development of a crossfunctional team responsible to implement the programme. Within this state, management needs to communicate its knowledge vision across the organisation, and make individuals aware to the KM programme and its expected benefits.

\subsection{High KM Infrastructure Capability}

The KM programme is in the state of high infrastructure capability when there is an emphasis on developing the infrastructure. During this stage the knowledge vision is translated into action by means of mission and value statements to encourage the growth of knowledge within the organisation. A knowledge culture of sharing and learning is promoted with individuals encouraged to participate and contribute. The organisation reviews its policies and processes, and implements systems of rewards and incentives to motivate and reward knowledge sharing behaviour. During this state information technology support is developed in the form repositories and collaborative technologies. Through the linkage provided by collaborative technologies the organisation attempts to integrate previously fragmented flows of knowledge, Teece (1998). Collaboration technologies are developed to allow individuals within the organisation to collaborate, thereby eliminating the structural and geographical impediments that may have previously prevented such interaction. Knowledge discovery technologies are developed to allow the organisation to find new knowledge that is either internal or external to the firm. Knowledge mapping and application technologies are developed to enable the firm to effectively track sources of knowledge, creating a catalogue of internal organisational knowledge, and apply its existing knowledge. An organisation's KM programme could be considered to be in this state when individuals have access to the above mentioned infrastructure but do not avail themselves of its complete potential or capability, due to the lack of practicing knowledge processes. 


\subsection{High KM Process Capability}

The KM programme can be considered to be in the state of high KM process capability when there is an emphasis on practicing knowledge processes. Openness and trust characterise the organisation's work environment and support knowledge sharing behaviours, which are included as an integral part of the training programmes. Communities of practice (Wenger and Snyder 2000) and centres of excellence evolve and individuals are encouraged to join and participate. Activities that establish an organisation's KM programme in a state of high knowledge process capability include identifying lessons learnt, best practices, benchmarking, brainstorming, group problem solving, mentoring and collaboration. The daily work processes support decision-making, feedback and interaction, which are made apparent in the team commitment. Knowledge champions from distributed centres meet regularly and knowledge flows across boundaries and development centres. Therefore an organisation would be in a state of high knowledge process capability and low infrastructure capability when the above mentioned knowledge processes are practiced but do not receive adequate support in the form of infrastructure support.

\subsection{Organisational State of KM}

An organisation will be in a state of organisational KM infrastructure and process capability when it achieves high availability of infrastructure capability to support frequent and regular practice of knowledge processes. In other words, knowledge processes are embedded in the daily routines, procedures and practices of the organisation which posses the knowledge infrastructure to support them. This state is characterised by a vibrant mix of vision, strategy, leadership, organisational structure, culture, technology infrastructure, and knowledge processes of creation, storage, retrieval, transfer, application and sharing. Forums such as communities of practice evolve and the organisational structure, culture, and technology support them. Lessons learnt are captured regularly and made available across the organisation, while best practices are implemented. Knowledge sharing and learning permeate the organisational environment of role models, mentoring, leadership, motivation, commitment, and training, where collaboration, feedback and interaction drive knowledge flow between individuals and teams. Acculturated knowledge champions and collaborative information technology support ensure that knowledge flows are not inhibited by organisational structures and distributed geographical locations, but instead flow across social networks and boundaries of the organisation. The knowledge flows ensure that the knowledge available within the organisation is current, integrated, usable, and applied. The organisation adopts a consistent approach to KM and it becomes a way of working within standardised work methods. Thus when KM is institutionalised within the organisation, the programme can be stated to be in the organisational state. 


\section{Conclusion}

This paper presents a KM Capability Framework based upon a case study that identified the knowledge management infrastructure and process capabilities required to support and facilitate knowledge management practices within a software project organisation. The paper analyses the development of these KM infrastructure and process capabilities from an initial state to an organisational state. The analysis established that two other intermediate states exist, and identified the possible paths an organisation's KM capabilities development might progress along, and discusses the activities and characteristics of each state through which the implementation of organisational KM programmes could possibly progress. By assessing and focusing on the KM infrastructure and process capabilities and their characteristics that are being developed and practiced, organisations can determine the current state of their KM programme implementation. Not all organisations will manage to progress to the organisational state of KM in one smooth journey, as observed in the XYZ case study. The framework presented in this paper enables organisations to analyse if their KM programme is more focused towards developing KM infrastructure capability rather than KM process capability, or whether limited KM infrastructure is available for the KM processes being practiced. The framework helps organisations to better understand the issues related to developing a KM initiative, as suggested by Eskerod and Skriver (2007), and analyse any imbalance that may exist and needs to be addressed. In doing so, the framework benefits organisations interested in making corrections and restoring the balance between KM infrastructure and process capability, thereby smoothening the path of successful KM implementation towards a state of organisational KM capability.

\section{References}

Alavi, M and Leidner, D.E, 'Knowledge Management and Knowledge Management Systems: Conceptual Foundations and Research Issues,' MIS Quarterly Vol 25 No 1, pp 107-136, March 2001.

Davenport, T.H and Prusak, L, 'Working Knowledge,' Harvard Business School Press, Boston, 1998.

DeLong, D, 'Building the Knowledge Based Organization: how Culture Drives Knowledge Behaviours,' Working Paper, Ernst \& Young's Centre for Business Innovation, Boston, 1997.

Eskerod, P and Skriver, H.J, 'Organizational Culture Restraining In-house Knowledge Transfer Between Project Managers-A Case Study,' Project Management Journal, vol. 38, no. 1, pp. 110-122, March 2007.

Gold, A.H, Malhotra, A, and Segars, A.H, 'Knowledge Management: An Organizational Capabilities Perspective,' Journal of Management Information Systems, Vol18, No 1, pp 185-214, Summer 2001.

Grant, R.M, 'Prospering in Dynamically-Competitive Environments: Organizational Capability as Knowledge Integration,' Organization Science Vol7 No 4, pp 375-387, July-August, 1996.

Ivers, J, 'Bringing Out Brilliance: Enabling Knowledge Creation in the Notes/Domino Environment, Enterprise Solutions, pp 24-27, November/December 1998. 
Khalifa, M, and Liu, V, Determinants of Successful Knowledge Management Programs, Electronic Journal on Knowledge Management, Vol 1, No 2, pp 103-112, 2003.

Leonard-Barton, D, 'Wellsprings of Knowledge: Building and Sustaining the Source of Innovation,' Harvard Business School Press, Boston, 1995.

Nonaka, I and Takeuchi, H, 'The Knowledge Creating Company: How Japanese Companies Create the Dynamics of Innovation,' Oxford University Press, New York, 1995.

O'Dell, C. and Grayson, C, "If only we knew what we know: Identification and transfer of internal best practices," California Management Review Vol. 40, No. 3, pp. 154-174, 1998.

Polanyi, M, 'The Tacit Dimension,' Routledge and Keon Paul, London, 1967.

Skyrme, D, and Amidon, D, 'New Measures of Success,' Journal of Business Strategy, pp 20-24, January/February 1998.

Spender, J.C, 'Making Knowledge the Basis of a Dynamic Theory of the Firm,' Strategic Management Journal Vol 17, pp 45-62, Special Issue 1996.

Styhre, A, 'Knowledge Management beyond codification: knowing as practice/concept,' Journal of Knowledge Management, Vol 7 No 5, pp 32-40, 2003.

Teece, D, 'Capturing Value from Knowledge Assets: the New Economy, Markets for KnowHow and Intangible Assets,' California Management Review, Vol 40, No 3, pp 55-79, 1998.

Wenger, E, and Snyder, W, 'Communities of Practice: The Organizational Frontier,' Harvard Business Review, Vol 78, No 1, pp 139-145, 2000. 